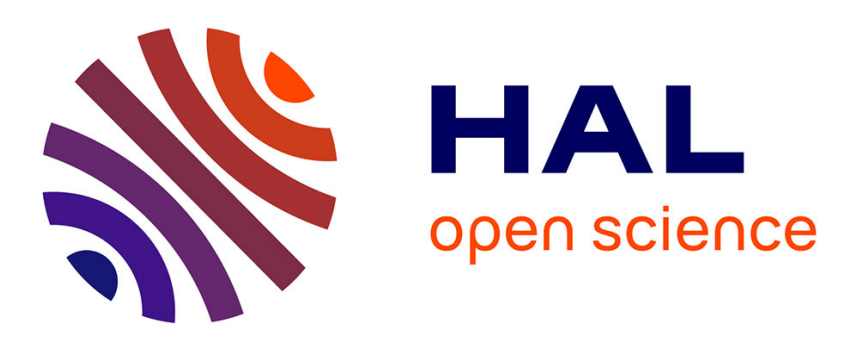

\title{
Modélisation de l'impact du respect des repères alimentaires du HCSP sur les apports nutritionnels des adultes français
}

\author{
Matthieu Maillot, Nicole Darmon
}

\section{To cite this version:}

Matthieu Maillot, Nicole Darmon. Modélisation de l'impact du respect des repères alimentaires du HCSP sur les apports nutritionnels des adultes français. JFN 2018, Journées Francophones de Nutrition, Nov 2018, Nice, France. 2018, JFN 2018 - Livre des résumés. hal-01947493

\section{HAL Id: hal-01947493 \\ https://hal.science/hal-01947493}

Submitted on 6 Dec 2018

HAL is a multi-disciplinary open access archive for the deposit and dissemination of scientific research documents, whether they are published or not. The documents may come from teaching and research institutions in France or abroad, or from public or private research centers.
L'archive ouverte pluridisciplinaire HAL, est destinée au dépôt et à la diffusion de documents scientifiques de niveau recherche, publiés ou non, émanant des établissements d'enseignement et de recherche français ou étrangers, des laboratoires publics ou privés. 


\section{Modélisation de l'impact du respect des repères alimentaires du HCSP sur les apports nutritionnels des adultes français*}

${ }^{1}$ MS-Nutrition, MARSEILLE, France

Matthieu Maillot ${ }^{1}$, Nicole Darmon ${ }^{2}$

2 MOISA, INRA, CIHEAM-IAMM, CIRAD, Montpellier SupAgro, 2 place Pierre Viala, 34060 Montpellier, France

\section{INTRODUCTION}

Le HCSP a rendu le 16 février 2017 un avis relatif à la révision des repères alimentaires pour les adultes du futur Programme national nutrition santé 2017-2021.

Par rapport aux repères actuellement en vigueur, on note :

i) l'ajout de nouveaux repères (légumineuses, charcuteries, fruits à coques, céréales complètes),

ii) une précision des repères sur les matières grasses (distinction des huiles riches en $\mathrm{ALA}$ ) et les viandes (distinction des viandes blanches et rouges),

iii) une modification du repère sur les produits laitiers qui passent de 3 à 2 portions/j.

\section{METHODES}

$\square$ Les repères du HCSP ont été traduits en repères chiffrés : quantités minimales et/ou maximales recommandées, en grammes ou en nombre de portions/j.

$\square$ Pour chacune des diètes observées des 1863 adultes de l'étude (>18ans) de l'enquête INCA2, l'approche de modélisation de diètes individuelles a été appliquée pour obtenir 2 nouvelles diètes (iso-énergétiques avec l'observée) respectant l'ensemble des repères du HCSP : I'une avec 2 portions/j (modèle HCSP) et l'autre avec 3 portions/j (modèle HCSP3) de produits laitiers.

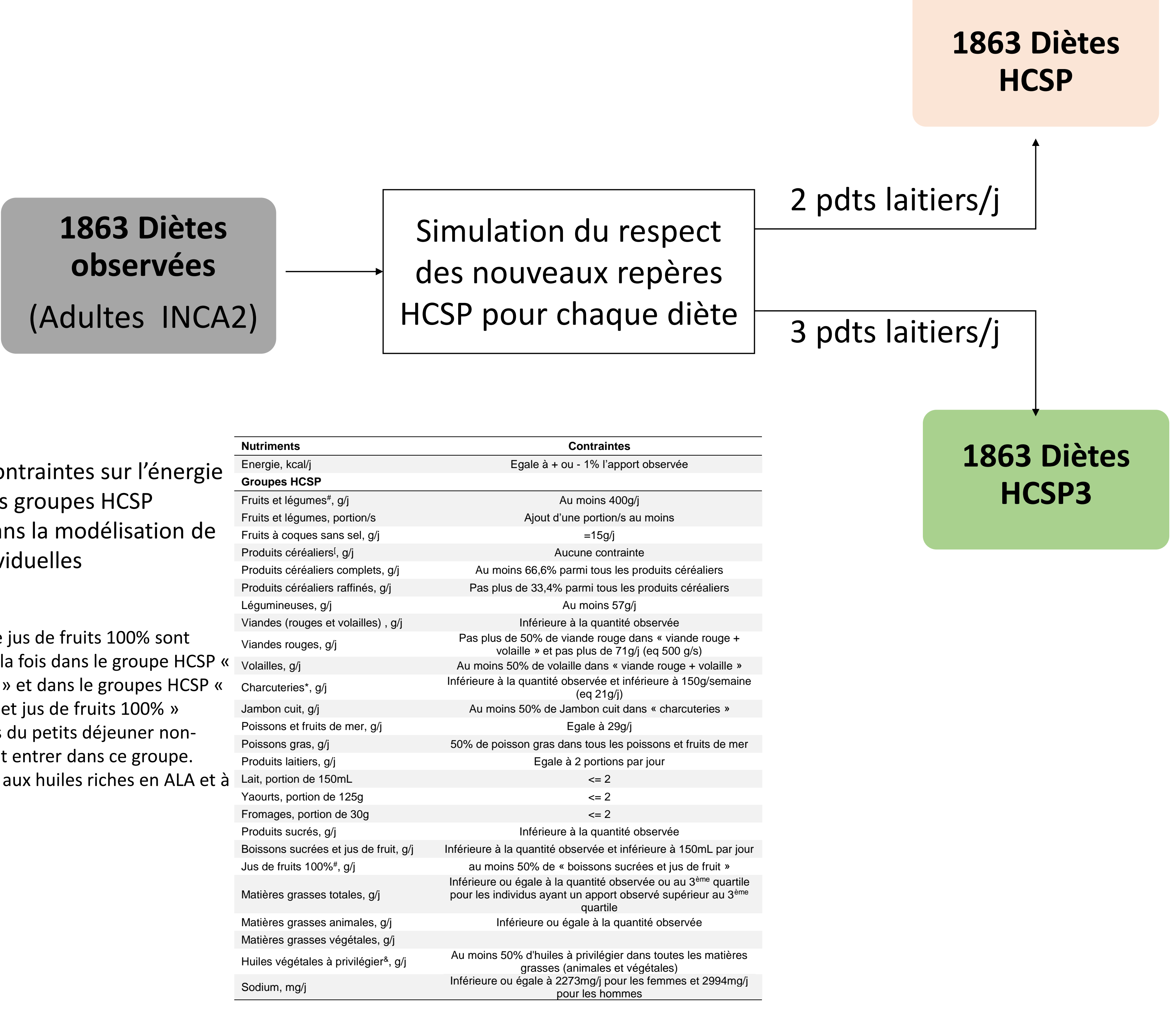

$\square$ La qualité nutritionnelle des diètes modélisées a été comparée à celle des diètes observées sur la base :

- des teneurs nutritionnelles moyennes,

- d'indicateurs de qualité globale (Densité Energétique-DE, Mean Adequacy Ratio-MAR, Mean Excess Ratio-MER) et

- du pourcentage d'inadéquation pour chaque nutriment (i.e., pourcentage de diètes ayant une teneur inférieure à la valeur nutritionnelle de référence, Besoin Nutritionnel Moyen ou Apport Satisfaisant).

\section{OBJECTIF}

L'objectif de cette étude était de modéliser, à partir des consommations individuelles INCA2, l'impact du respect des nouveaux repères de consommation du HCSP sur la composition nutritionnelle des diètes, et de comparer cet impact selon que le repère pour les produits laitiers est fixé à 2 ou à 3 portions/j.

\section{CONCLUSION}

$\rightarrow$ Le respect des repères HCSP améliore la qualité nutritionnelle globale mais dégrade les teneurs en calcium, vit B2, vit B12, et EPA+DHA.

$\rightarrow$ Le respect des repères HCSP avec $3 p / j$ de produits laitiers permettrait d'atteindre la même qualité nutritionnelle globale qu'avec $2 p / j$ mais permettrait, en plus, de réduire considérablement le pourcentage d'inadéquation d'apports en calcium et vit B2

\section{RESULTATS}

$\square$ Quel que soit le modèle, il a été possible de construire une diète modélisée respectant l'ensemble des repères pour $98 \%$ des diètes.

$\square$ La qualité nutritionnelle globale des diètes simulées HCSP et HCSP3 est améliorée :
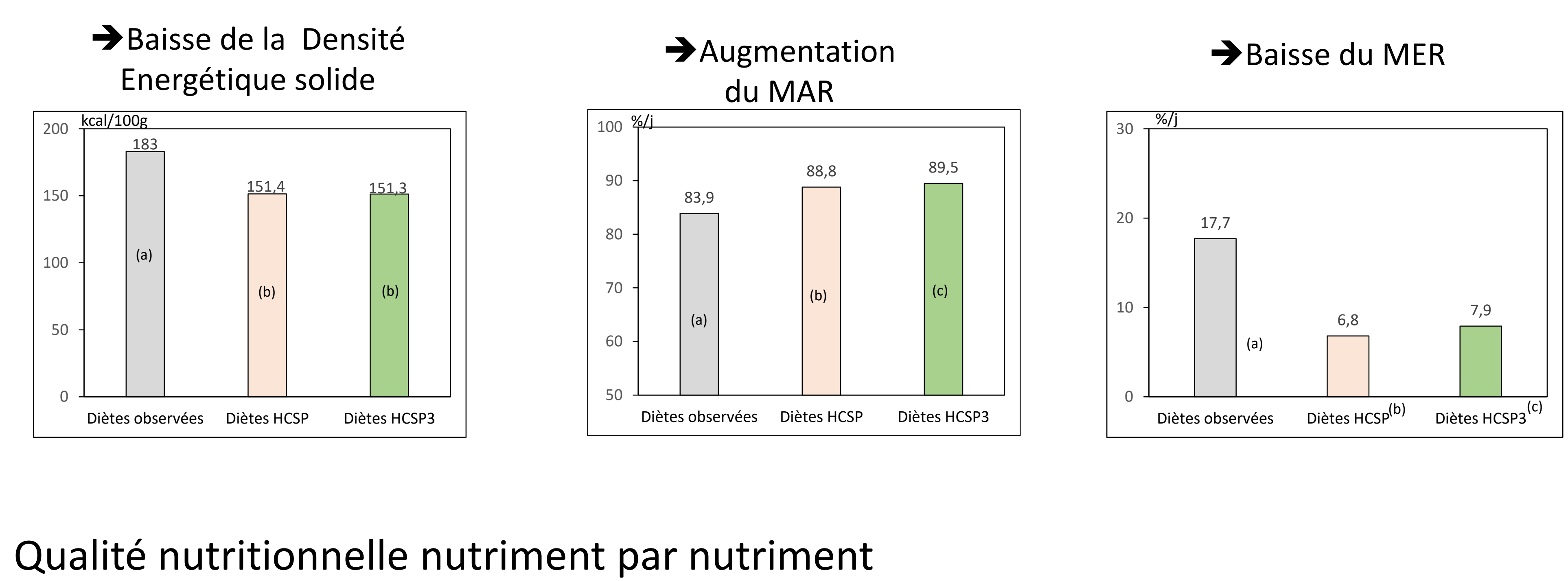

$\square$ Qualité nutritionnelle nutriment par nutriment

Pourcentage d'inadéquation (\%) au Besoin Nutritionnel Moyen ou à l'Apport Satisfaisant

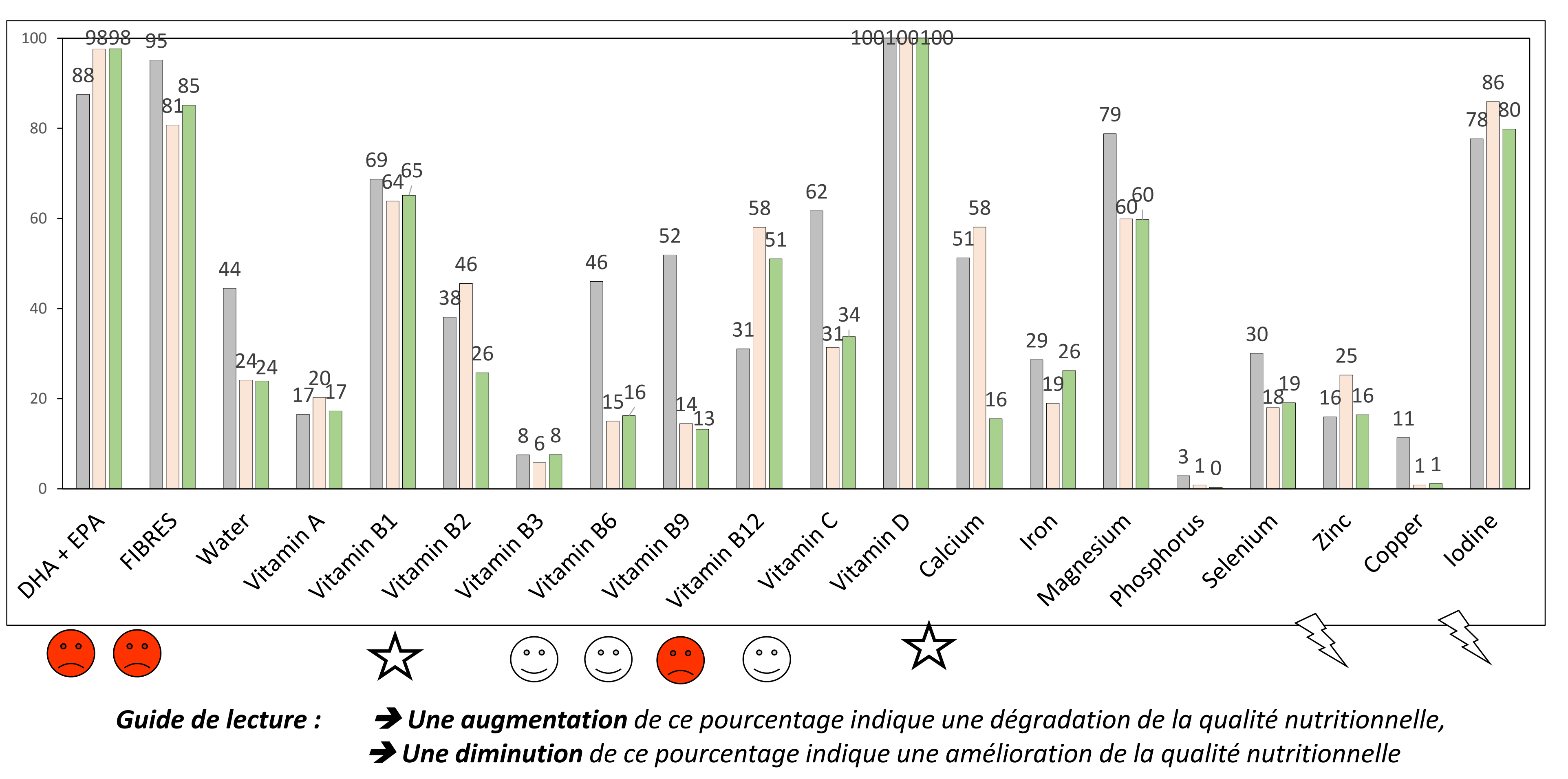

- Les vit. C, B9 et B6 étaient les nutriments les plus améliorés par les 2 modèles

产 Pour la vitamine B2 et le calcium, le modèle HCSP dégradait la situation alors que le modèle HCSP3 l'améliorait (38\% ; $46 \%$ et $26 \%$ d'inadéquation pour la vitamine B2 et 51 ; $58 \%$ et $16 \%$ pour le calcium).

Le modèle HCSP dégradait la situation pour le zinc et l'iode, alors que le HCSP3 ne la dégradait pas pour le zinc et la dégradait moins pour l'iode.

Quel que soit le modèle, le pourcentage d'inadéquation restait très élevé (supérieur à $80 \%$ ) pour les fibres, les acides gras oméga-3 à longue chaine EPA+DHA et la vitamine D. La vitamine B12 était dégradée par les 2 modèles. 